\title{
Ultrashort peptides: minimum number in amino acid residues, maximum number in bioapplications.
}

U Ltra-short peptides are an intrigue new type of biomaterials for various bioapplications. Over the decades, peptides have evolved as biomaterials due to the ease of synthesis, ease of scaling up, feasibility to be functionalized/modified, and recognition by biological system ${ }^{1}$. Peptide-based hydrogels are widely used as scaffolds for encapsulation and delivery of drugs, genes and cells. They are made of natural amino acids. Thus they are non-immunogenic. They contain carboxylic and amine groups. Thus they can be easily functionalized with bioactive moieties. However, for peptides with more than 16 amino acid residues, they are expensive due to the high synthesis cost. As such, ultra-short peptides with only 2 to 7 amino acid residues stand out due to their easy and cost-effective synthesis, facile assembly in aqueous solution, biocompatibility and mechanical stability. These ultrashort peptides can form hydrogels without the addition of enzymes or other types of cross-linkers.

The discovery of these ultrashort peptides dates back to 2011. Dr. Charlotte Hauser (at that time she and I were both working at Institute of Bioengineering and Nanotechnology in Singapore) found a novel class of ultrashort linear peptide with 2 to 7 natural aliphatic amino acids ${ }^{2}$. These peptides are amphiphilic, consisting of an aliphatic amino acid tail of decreasing hydrophobicity and a hydrophilic head. These peptides self-assemble most likely via parallel-antiparallel a-helical pair formation and subsequent stacking into fibers that condense to b-turn fibrils. Fibrils further aggregate and form nanofibrous scaffolds - macroscopic hydrogels. Under scanning electron microscope, we can clearly observe nanofibrous structure at nanoscale, and honeycomb structure at microscale for this type of peptide material. The nanofibrous scaffold is capable of entrapping more than $99 \%$ water, gelation even at $1 \mathrm{mg} / \mathrm{mL}$. These ultrashort peptides are thermally stable. They were tested with two commonly used sterilization methods in lab, UV radiation and autoclave. From NMR spectra and DSC curves, these peptides showed no different from untreated samples. Then these peptides were examined with three types of mammalian cells, including human mesenchymal stem cells, porcine nucleus pulpous cells and rabbit pigment epithelial cells. Cell culture results showed that these peptides were noncytotoxic and nongenotoxic ${ }^{3}$. With the preliminary data of biocompatibility for these ultrashort peptides, researchers started pursuing different bioapplications as follow:

1. Ultrashort peptides as cell culture scaffold

2. Ultrashort peptides as 3D bioprinting inks

3. Ultrashort peptides as drug delivery vehicles

4. Ultrashort peptides as bioimaging probes

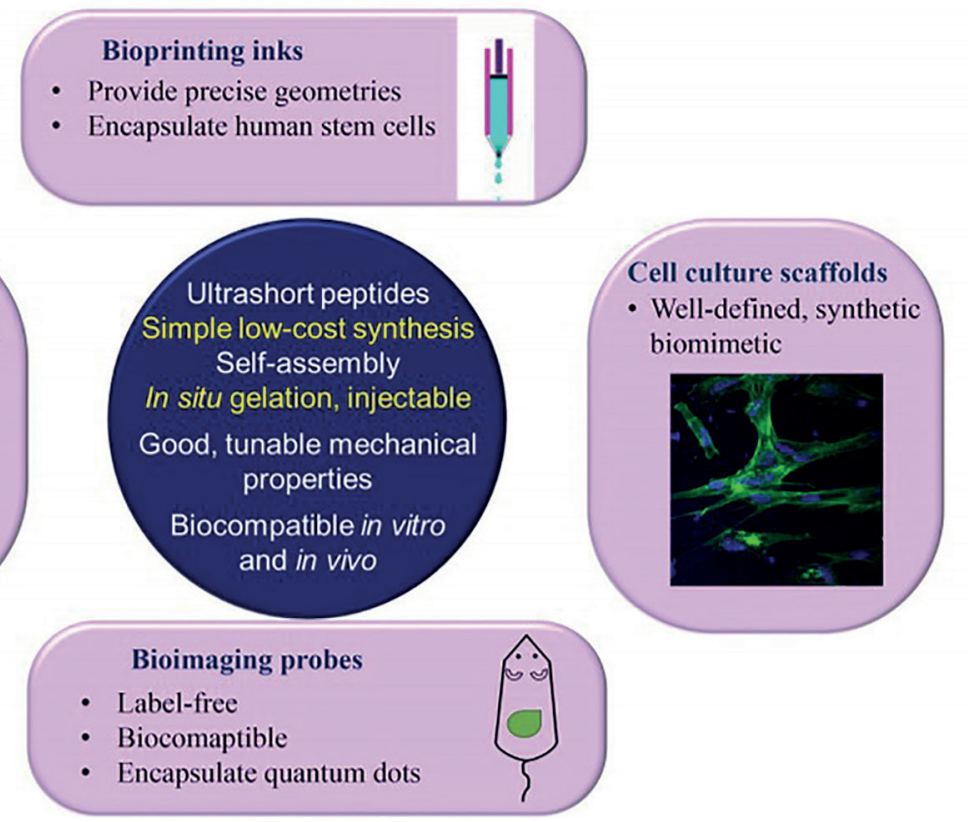

Figure 1. Bioapplications of ultra-short peptides as cell culture scaffolds, drug delivery vehicles, bioprinting inks and bioimaging probes. 
1. Ultrashort peptides as cell culture scaffold Ultrashort peptides mimic extracellular matrix (ECM). ECM proteins, such as fibronectin and laminin, are commonly applied as surface coatings to improve cell adhesion. Amino acid sequences of RGD and YIGSR are derived from fibronectin and laminin. Instead of immobilizing the whole protein, researchers prefer to immobilize short peptides on the cell culture substrates as it is easy to control these peptides' orientation on culture substrate at a lower cost.

2. Ultrashort peptides as 3D bioprinting inks 3D bioprinting enable us to create scaffolds with precise geometries. In 2015, Loo et al. ${ }^{4}$ reported using ultrashort peptide hydrogels as bioink for 3D bioprinting. These peptide hydrogels undergo instantaneous gelation under physiological conditions, resulting in peptide hydrogel scaffolds. Furthermore, the hydrogel scaffolds support long-term 3D cultures of encapsulated human stem cells, as well as primary cells. The cells were further differentiated and grew into organotypic structures (skin and small intestine), which could be a useful platform for drug screening and diagnostics.

3. Ultrashort peptides as drug delivery vehicles

Drug delivery basically faces two hurdles: targeting both in vitro and in vivo. Ultrashort peptides are a perfect choice in this regard. Reithofer et al. ${ }^{5}$ applied "click chemistry" to conjugate anti-cancer drug to ultrashort peptides. These chemically modified ultrashort peptides can form hydrogel with pristine peptides. It was used to treat breast cancer locally in an injectable form. Significant tumor growth inhibition was observed in a mice model.

4. Ultrashort peptides as bioimaging probes

Bioimaging refers to any imaging technique used in life sciences. It uses light, fluorescence, electrons, X-rays, ultra-sound, magnetic resonance as sources for imaging ${ }^{6}$. Quantum dots (QDs) are commonly use as fluorescent probes due to their superior brightness, and long fluorescent lifetimes ${ }^{7}$. However, QDs are highly toxic due to due to their content of heavy metal elements. Biocomaptible ultrashort peptides can be used to encapsulate QDs, providing a protective layer. Second harmonic generation (SHG) is a label-free imaging technique, offering many advantages over conventional fluorescent imaging technique ${ }^{7}$. Aromatic ultrashort peptides, such as FF and FFF, and aliphatic ultrashort peptides, such as IVD, are SHG-active, which make them suitable as bioimaging probes ${ }^{6}$.

In this editorial article, we discussed ultrashort peptides ( 2 to 7 amino acids) and their bioapplications. They can form various types of nanostructures and possess interesting electrical, mechanical and optical properties. These ultrashort peptides can be applied as bioimaging probes, bioprinting ink, cell culture scaffolds, and drug delivery vehicles.

\section{References}

1. M. Ni, C.A.E. Hauser, Self-Assembled Peptide Nanostructures for Regenerative Medicine and Biology, (2015) 63-90.

2. C.A. Hauser, R. Deng, A. Mishra, Y. Loo, U. Khoe, F. Zhuang, D.W. Cheong, A. Accardo, M.B. Sullivan, C. Riekel, J.Y. Ying, U.A. Hauser, Natural tri- to hexapeptides self-assemble in water to amyloid beta-type fiber aggregates by unexpected alpha-helical intermediate structures, Proc Natl Acad Sci U S A 108(4) (2011) 1361-6.

3. A. Mishra, Y. Loo, R. Deng, Y.J. Chuah, H.T. Hee, J.Y. Ying, C.A.E. Hauser, Ultrasmall natural peptides self-assemble to strong temperature-resistant helical fibers in scaffolds suitable for tissue engineering, Nano Today 6 (2011) 232-239.
4.Y. Loo, A. Lakshmanan, M. Ni, L.L. Toh, S. Wang, C.A. Hauser, Peptide Bioink: Self-Assembling Nanofibrous Scaffolds for Three-Dimensional Organotypic Cultures, Nano Lett 15(10) (2015) 691925.

5. M.R. Reithofer, K.-H. Chan, A. Lakshmanan, D.H. Lam, A. Mishra, B. Gopalan, M. Joshi, S. Wang, C.A.E. Hauser, Ligation of anti-cancer drugs to self-assembling ultrashort peptides by click chemistry for localized therapy, Chem. Sci. 5 (2014) 625-630.

6. M. Ni, S. Zhuo, Applications of self-assembling ultrashort peptides in bionanotechnology, RSC Advances 9(2) (2019) 844-852.

7. M. Ni, S. Zhuo, Nonlinear optical microscopy: Endogenous signals and exogenous probes, Annalen der Physik 527(7-8) (2015) 471489.

School of Biological Sciences \& Engineering, Yachay Tech University, Hacienda San José s/n, San Miguel de Urcuquí 100105, Ecuador 